\title{
可见光与红外融合的汽车抗晕光图像评价方法
}

\author{
柴改霞，郭全民，孙晓娟 \\ (西安工业大学 电子信息工程学院, 陕西 西安 710021)
}

\begin{abstract}
摘要: 为了解决汽车抗晕光场景中, 融合图像质量的客观评价与人眼视觉效果不一致的问题, 提出 了一种新的可见光与红外融合图像质量评价方法, 该方法通过设计自适应迭代阈值法自动确定融合 图像的晕光临界灰度值, 并将融合图像自动分为晕光区和非晕光区。针对晕光区, 设计晕光消除度 指标评价晕光消除的效果; 针对非晕光区, 从多方面评价色彩、细节信息的增强效果，并甄选出合 适的指标构成完整的图像质量评价体系。为验证该方法的合理性，对采用 4 种不同算法的融合图像 进行评价, 实验分析表明, 该方法的主客观评价结果一致, 适于评判不同可见光与红外融合的抗晕 光图像质量及算法的优劣。
\end{abstract}

关键词: 抗晕光; 图像融合; 质量评价; 自动分区

中图分类号：TP391.4 文献标识码：A 文章编号：1001-8891(2020)04-0378-07

\section{Vehicle Anti-halation Image Evaluation Method Based on Visible and Infrared Fusion}

\author{
CHAI Gaixia， GUO Quanmin， SUN Xiaojuan \\ (School of Electronic Information Engineering, Xi'an Technological University, Xi'an 710021, China)
}

\begin{abstract}
Owing to the problem that objective evaluation of fusion image quality is inconsistent with human visual effects in vehicle anti-halation, a new quality evaluation method is proposed in this paper for visible and infrared fusion images. The method can automatically determine halo critical gray values to divide fusion images into halo and no-halo regions by designing an adaptive iterative threshold algorithm. For the halo region, the halo limination effect is evaluated by designing a halo limination index. For the no-halo region, enhancement effects of color and detail information are evaluated from various aspects. Appropriate indexes are selected to constitute a complete image quality evaluation system. Fusion images of four different algorithms are evaluated to verify their rationality. Experimental analysis show that subjective and objective evaluation results of this method are consistent, rendering it suitable for evaluating anti-halation image quality and the algorithm of different visible and infrared fusion.
\end{abstract}

Key words: anti-halation, image fusion, quality evaluation, automatic dividing

\section{0 引言}

夜间会车时, 滥用远光灯使驾驶员感到非常刺 眼, 无法看清行车路线及行人, 极易导致严重的交通 事故 ${ }^{[1]}$ 。因此, 研究汽车抗晕光有重要意义。传统的 光学原理抗晕光方法使暗处信息难以观察 ${ }^{[2]}$; 红外夜 视仪的图像无色彩、细节信息缺失严重 ${ }^{[3-5]}$; “排洪” 面阵 CCD 图像传感器把高亮度的多余电荷排出存储 势阱, 仅降低了晕光的亮度 ${ }^{[6]}$; 光积分时间不同的两 路可见光融合依然存在晕光现象 ${ }^{[7]}$ 。可见光与红外融
合的抗晕光方法 ${ }^{[8]}$ 结合可见光图像色彩细节信息丰 富、红外图像无晕光的优点, 为解决夜间行车的晕光 问题提供了一种新途径, 具有较好的应用前景, 但现 有的无参考、全参考和基于视觉系统的评价方法都不 能准确地评价抗晕光融合图像的质量 ${ }^{[9]}$ 。

其中, 无参考图像评价方法通过客观指标评价融 合图像自身质量 ${ }^{[10]}$ 。融合图像消除晕光后, 晕光部位 的高亮度信息被剔除, 导致反映亮度的均值降低; 晕 光消除越彻底, 明暗对比度越低, 晕光处的纹理细节 反差越小, 标准差和边缘强度越低。全参考图像评价 
方法评价融合图像对原始图像的信息保留度 ${ }^{[1]}$ 。例如 交叉熵, 在融合图像非晕光部位, 该指标能很好地反 映其对原始图像细节信息的保留度, 但在晕光部位, 晕光消除越彻底, 与可见光图像相似度越低。基于视 觉系统的评价方法考虑人眼的视觉效果 ${ }^{[12]}$ 。如边缘保 持度, 在融合图像非晕光部位, 该指标能很好地反映 其对原始图像边缘、轮廓的保留度, 但在晕光部位, 军光消除越彻底, 军光边缘、轮廓等信息保留越少, 结构相似度也存在同样的问题。以上指标均出现晕光 消除越彻底, 融合图像质量的客观评价结果越差, 与 视觉效果不统一的现象。

针对上述问题, 本文提出一种可见光与红外融合 的汽车抗晕光图像评价方法: 通过设计自适应迭代阈 值法实现融合图像的自动分区, 对晕光区采用设计的 晕光消除度指标衡量晕光消除的效果; 对非晕光区从 多角度评价色彩、细节信息的增强效果, 并甄选合适 的指标构成完整的评价体系, 全面、合理地评判汽车 抗晕光融合图像的质量及算法的优劣。

\section{1 可见光与红外融合的抗晕光图像评价原理}

汽车抗晕光图像融合的目的, 与一般图像融合不 同, 首要是消除高亮的晕光, 再次是增强暗处的色彩、 细节信息。图 1(a)可见光图像色彩丰富、车牌较清晰, 但存在明显的晕光现象, 车辆轮廓、道路边缘、背景 建筑及行人难以观察; 图 1(b)红外图像无晕光, 轮廓 细节清晰, 但色彩和车牌缺失; 图 1(c)采用改进 IHS-Curvelet 变换 ${ }^{[13]}$ 得到无晕光、轮廓细节清晰、色 彩丰富的融合图像。

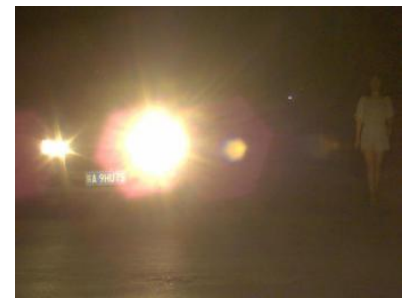

(a)可见光图像

(a) Visible image

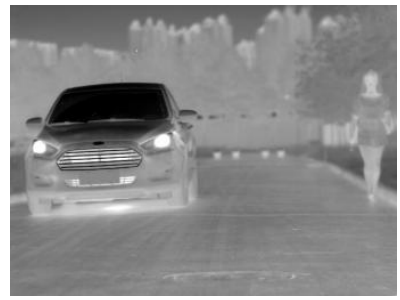

(b) 红外图像 (b) Infrared image

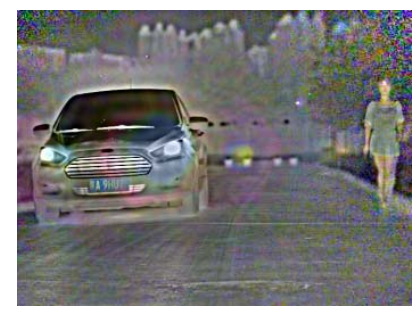

(c) 融合图像(c) Fusion image

图 1 可见光、红外及其融合图像

Fig.1 Visible, infrared and fusion image
汽车抗晕光融合图像同一般可见光与红外融合 图像相比存在 3 点显著差异: (1)融合图像消晕光后与 可见光图像差异显著, 晕光消除越彻底差异越大; (2) 可见光图像的光晕梯度及明暗边界十分明显, 晕光消 除越彻底, 融合图像原晕光部位的梯度、边界越不明 显; (3)可见光图像晕光部位的高亮度造成其他部位的 亮度更暗, 细节信息更不易观察。

晕光区的高亮度使客观评价不能真实地反映融 合图像的质量, 为避免晕光干扰, 将融合图像的晕光 和非晕光区分开评价。在晕光区，根据融合图像与可 见光、红外图像的相似度评判军光消除的效果。与红 外图像越相似, 晕光消除越彻底, 反之晕光消除越不 彻底。非晕光区图像不存在晕光干扰, 采用现有的方 法评价色彩、细节信息的增强效果。

\section{2 抗晕光图像评价方法实现}

根据可见光图像的晕光程度自动确定可见光灰 度图的晕光临界灰度值, 实现融合图像的自动分区。 针对晕光与非军光区图像的处理目标不同, 晕光区设 计晕光消除度指标衡量融合图像晕光消除的效果, 非 晕光区从多角度评价色彩、细节信息的增强效果。

\section{1 抗晕光融合图像的自动分区}

2.1.1 确定晕光临界灰度值

由于晕光区的灰度值明显高于非晕光区, 根据晕 光区与非军光区交接处的灰度值划分两区域, 即确定 晕光临界灰度值 $G_{\mathrm{c}}$ 。自适应迭代阈值法: 以图像灰度 中值作为初始阈值 $T_{0}$;

$$
T_{0}=0.5\left(\mathrm{In}_{\max }+\mathrm{In}_{\min }\right)
$$

式中: $\mathrm{In}_{\text {max }} 、 \mathrm{In}_{\min }$ 分别是像素的最大、最小灰度值。 利用阈值 $T_{i}$ 将图像分为两区域 $R_{1}$ 和 $R_{2}$, 计算 $R_{1}$ 和 $R_{2}$ 的灰度均值 $\mu_{1}$ 和 $\mu_{2}$;

$$
\mu_{1}=\frac{1}{L_{1}} \sum_{j=0}^{L_{1}-1} \operatorname{In}(j), \quad \mu_{2}=\frac{1}{L_{2}} \sum_{j=L_{1}}^{L-1} \operatorname{In}(j)
$$

式中: $\operatorname{In}(j)$ 是第 $j$ 个像素的灰度值; $L$ 为图像总像素数; $L_{1} 、 L_{2}$ 分别为区域 $R_{1} 、 R_{2}$ 像素数, $L=L_{1}+L_{2}$ 。计算 新的阈值 $T_{i+1}$;

$$
T_{i+1}=m\left(\mu_{1}+\mu_{2}\right)
$$

式中: $m$ 为图像晕光区的分割效果满足人眼视觉效果 的自适应系数, 随晕光程度自动调节。

重复公式(2)(3), 待阈值不再变化时迭代结束, 最 新得到的阈值为图像的晕光临界灰度值 $G_{\mathrm{c}}$ 。

\subsection{2 确定自适应系数}

晕光区高亮度的信息量影响整幅图像的灰度值, 晕光信息量与晕光面积有关, 则晕光面积间接影响图 
像的灰度值。研究两车会车时的晕光图像, 采集不同 类型道路车辆由远及近, 晕光面积从小到大再变小的 大量晕光图像, 获得自适应系数 $m$ 、晕光区与非晕光 区面积比 $s$ 的对应点集 $\left(s_{i}, m_{i}\right)$, 研究发现面积比 $s$ 和自 适应系数 $m$ 的关系接近 $m=a \times s^{b}+c$ 的递减函数, 利 用非线性最小二乘法拟合曲线:

$$
Q=\sum_{k=1}^{N}\left[y_{k}-f\left(x_{k}, \theta\right)\right]^{2}
$$

式中: $x 、 y$ 分别是系统的输入和输出; $f$ 是参数 $\theta$ 的非 线性模型; $Q$ 达到极小值时得参数估计值。

当 $a=-0.6701, b=0.07411, c=1.175$ 时, 自适 应系数 $m$ 的取值使融合图像晕光区的分割效果满足 人眼视觉效果, 如下式:

\subsection{3 自动分区}

$$
m=-0.6701 \times s^{0.07411}+1.175
$$

由军光临界灰度值 $G_{\mathrm{c}}$ 划分两区域, 灰度值大于 等于 $G_{\mathrm{c}}$ 的像素构成军光区 $A_{\mathrm{H}}$, 反之为非军光区 $A_{\overline{\mathrm{H}}}$ :

$$
\left\{\begin{array}{l}
A_{\mathrm{H}}=\left\{Y(i, j) \mid Y(i, j) \geq G_{\mathrm{c}}\right\} \\
A_{\overline{\mathrm{H}}}=\left\{Y(i, j) \mid Y(i, j)<G_{\mathrm{c}}\right\}
\end{array}\right.
$$

将图 1 中的原始图像和融合图像进行分区, 如图 2。

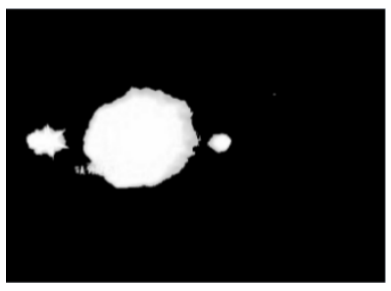

(a) 可见光图像晕光区

(a) Halo region of visible image

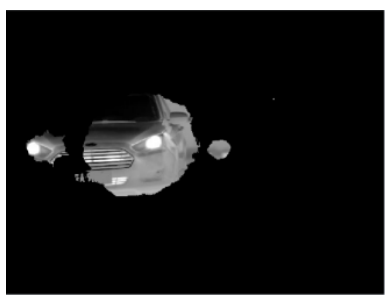

(c) 红外图像晕光区

(c) Halo region of infrared image

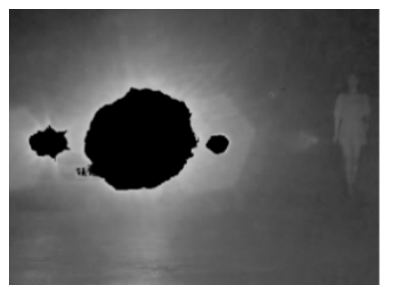

(b)可见光图像非晕光区

(b) No-halo region of visible image

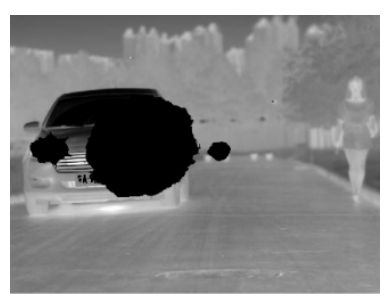

(d)红外图像非晕光区

(d) No-halo region of infrared image

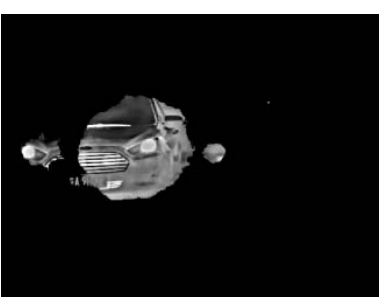

(e) 融合图像晕光区

(e) Halo region of fusion image

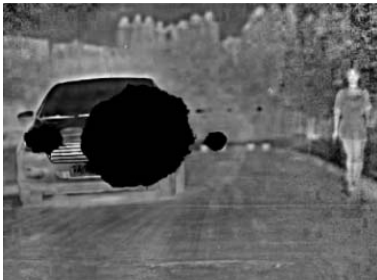

(f)融合图像非晕光区

(f) No-halo region of fusion image
图 2 自动分区图像 Fig.2 Automatic division images

\section{2 晕光消除度}

为评价融合图像的晕光消除效果, 以融合图像晕 光区与可见光、红外图像的接近程度为参考, 考虑人 眼视觉感知特点和图像的结构信息, 综合亮度、对比 度、结构 3 方面表征融合图像与原图的相似度, $l_{R-\mathrm{FU}}$, $C_{R-\mathrm{FU}}, S_{R-\mathrm{FU}}$ 分别为原图与融合图像的亮度相似度、对 比度相似度、结构相似度:

$$
\begin{gathered}
l_{R-\mathrm{FU}}=\frac{2 \bar{x}_{R} \bar{x}_{\mathrm{FU}}+C_{1}}{\bar{x}_{R}^{2}+\bar{x}_{\mathrm{FU}}^{2}+C_{1}} \\
c_{R-\mathrm{FU}}=\frac{2 \sigma_{R} \sigma_{\mathrm{FU}}+C_{2}}{\sigma_{R}^{2}+\sigma_{\mathrm{FU}}^{2}+C_{2}} \\
S_{R-\mathrm{FU}}=\frac{\sigma_{R-\mathrm{FU}}+C_{3}}{\sigma_{R} \sigma_{\mathrm{FU}}+C_{3}}
\end{gathered}
$$

式中: $R$ 为参考图像, $R \in\{\mathrm{VI}, \mathrm{IR}\}, \mathrm{VI} 、 \mathrm{IR} 、 \mathrm{FU}$ 分 别为可见光、红外、融合图像; $\bar{x} 、 \sigma$ 分别为图像的 均值、标准差; $\sigma_{R-\mathrm{FU}}$ 为参考与融合图像的协方差; $C_{1} 、 C_{2} 、 C_{3}$ 为常数, 避免分母为 0 。设定 $C_{3}=C_{2} / 2$, 原图与融合图像的相似度指标 $S_{R-F U}$ 可表示为:

$$
\begin{aligned}
& S_{R-\mathrm{FU}}=l_{R-\mathrm{FU}} \cdot c_{R-\mathrm{FU}} \cdot S_{R-\mathrm{FU}}= \\
& \frac{\left(2 \bar{x}_{R} \bar{x}_{\mathrm{FU}}+C_{1}\right)\left(2 \sigma_{R-\mathrm{FU}}+C_{2}\right)}{\left(\bar{x}_{R}^{2}+\bar{x}_{\mathrm{FU}}^{2}+C_{1}\right)\left(\sigma_{R}^{2}+\sigma_{\mathrm{FU}}^{2}+C_{2}\right)}
\end{aligned}
$$

根据式(10)将融合图像与红外、可见光图像的相 似度作差后进行归一化, 构造晕光消除度 $D$ :

$$
D=0.5 \times\left[\frac{\left(2 \bar{x}_{\mathrm{IR}} \bar{x}_{F \mathrm{U}}+C_{1}\right)\left(2 \sigma_{\mathrm{IRFU}}+C_{2}\right)}{\left(\bar{x}_{\mathrm{IR}}^{2}+\bar{x}_{\mathrm{FU}}^{2}+C_{1}\right)\left(\sigma_{\mathrm{IR}}^{2}+\sigma_{\mathrm{FU}}^{2}+C_{2}\right)}-\frac{\left(2 \bar{x}_{\mathrm{VI}} \bar{x}_{F \mathrm{U}}+C_{1}\right)\left(2 \sigma_{\mathrm{VIFU}}+C_{2}\right)}{\left(\bar{x}_{\mathrm{VI}}^{2}+\bar{x}_{F U}^{2}+C_{1}\right)\left(\sigma_{\mathrm{VI}}^{2}+\sigma_{\mathrm{FU}}^{2}+C_{2}\right)}+1\right]
$$

式中: $D$ 的取值范围为 $[0,1], D$ 越接近 1, 融合图与红外图像越接近, 晕光消除越彻底。

\section{3 非晕光区色彩、细节增强的评价指标}

非晕光区没有晕光信息的干扰，用现有的评价方 法 ${ }^{[14-15]}$ 从融合图像自身特性、融合图像对原始图像信 息的保留度和人眼视觉效果 3 方面评价融合图像的质
量, 并通过实验对比与分析, 甄选出合适的指标, 准 确、合理地评判融合图像质量及算法优劣。

1) 融合图像自身特性方面。计算非晕光区融合 图像的均值 $\bar{X}$ 、标准差 $\sigma$ 、信息熵 $E$ 、平均梯度 (average 
gradient, AG)、边缘强度 (edge intensity, EI)、空间频 率 (spatial frequency, SF) 等。

2) 融合图像对原始图像信息的保留度方面。计 算峰值信噪比 (peak signal to noise ratio, PSNR)、交 叉熵 (cross entropy, CE)、互信息 (mutual information, $\mathrm{MI}$ )、均方根误差（root mean square error, RMSE）等 指标。

3) 人眼视觉效果方面。计算结构相似度 (structure similarity index, SSIM）和边缘保持度 $Q^{\mathrm{AB} / \mathrm{F}}$ 。

\section{3 实验及结果分析}

为验证本文的评价方法能全面、合理地评判汽车 抗晕光融合图像的晕光消除效果, 色彩、细节信息增 强效果, 以 HIS (intensity, hue, saturation) 变换、 Curvelet 变换、IHS-Curvelet 变换、改进 IHS-Curvelet 变换的融合图像为例进行评价, 图 3 为市郊道路对向 来车开启远光灯约 $10 \mathrm{~m}$ 处的 4 种算法融合结果。

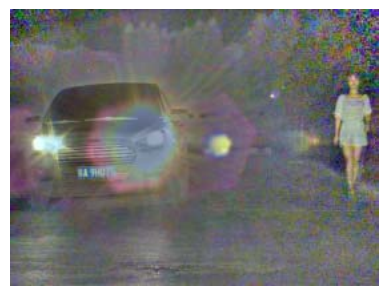

(a) IHS 变换

(a) IHS transform

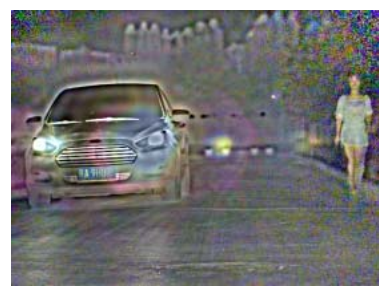

(c) IHS-Curvelet 变换

(c) IHS-Curvelet transform

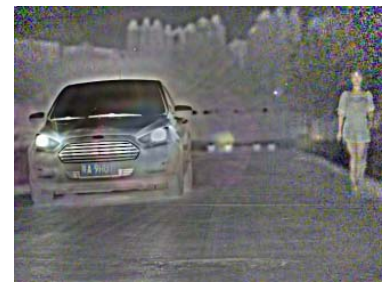

(b) Curvelet 变换

(b) Curvelet transform

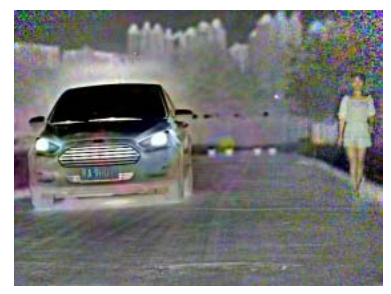

(d) 改进 IHS-Curvelet 变换

(d) Improved IHS-Curvelet
图 3 不同算法的融合图像

Fig.3 Fusion image of different algorithms

\section{1 主观评价结果}

选 10 位擅长图像处理的观察者从晕光消除度、 边缘轮廓、色彩细节 3 方面评判融合图像的质量。

图 3(a)IHS 变换的晕光严重, 车辆轮廓、车牌、 道路边缘及背景建筑模糊, 但色彩丰富; 图 3(b) Curvelet 变换的晕光较少, 细节较清晰, 但缺失色彩。 整体上 Curvelet 变换的图像质量优于 IHS 变换。

图 3(c)IHS-Curvelet 较图 3(a), 晕光消除度和清晰 度明显改善, 色彩无差别, 在 IHS 变换中融入 Curvelet 变换能明显改善图像的质量; 图 3(c)较图 3(b), 晕光
消除度略差, 清晰度无差别, 但色彩丰富。

图 3(d)改进 IHS-Curvelet 较图 3(c), 晕光基本完 全消除, 清晰度更好, 色彩无差别, 图像质量更优。

\section{2 客观评价结果}

为避免主观意识干扰，结合客观指标从两方面进 行评判。晕光区由晕光消除度 $D$ 评价晕光消除的效 果; 非晕光区从多角度评价色彩、细节信息的增强效 果, 甄选合适的指标构成完整的质量评价体系。

\subsection{1 晕光区域评价结果}

计算 4 种融合图像的晕光消除度 $D$ 如表 1 。

$$
\text { 表 } 1 \text { 晕光消除度 }
$$

Table 1 Eliminated degree of halo

\begin{tabular}{lc}
\hline \multicolumn{1}{c}{ Algorithm } & $D$ \\
\hline IHS & 0.6579 \\
Curvelet & 0.7683 \\
IHS-Curvelet & 0.8200 \\
Improved IHS-Curvelet & 0.9001 \\
\hline
\end{tabular}

从表 1 看出, IHS 变换的晕光消除效果最差, 因 IHS 变换的光谱分辨率低, 使融合图像与原图差异较 大; Curvelet 较 IHS 变换高 $16.7 \%$, 晕光消除效果更 好, 因 Curvelet 变换的各向异性, 能更多地保留图像 的细节信息，与原图结构差异更小。

IHS-Curvelet 较 IHS 变换提高 $25 \%$, 因其将 IHS 空间下的亮度和色彩分量分开处理, 避免了光谱扭曲 现象, 在 IHS 变换中融入 Curvelet 变换能提高 IHS 变 换的稳定性; IHS-Curvelet 较 Curvelet 变换提高 7\%, 因 IHS 变换丰富了图像的色彩, 在 Curvelet 变换中融 入 IHS 变换能略微提高图像的质量。综上, IHS-Curvelet 比两种单一变换的晕光消除效果好。

改进 IHS-Curvelet较改进前提高 9.8\%, 效果更好, 因改进的低频系数权值自动调节融合策略 ${ }^{[16]}$ 能剔除 所有晕光信息, 避免其参与融合过程。

\subsection{2 非晕光区域评价结果}

对非晕光区从以下 3 方面评价融合图像的质量。

1) 融合图像自身特性方面。为验证本文方法的 有效性，与不分区数据进行对比，如表 2 所示。

从表 2 看出, 4 种算法不分区的各项指标均比非 晕光区大, 由于高亮度晕光信息的存在，使不分区融 合图像的均值变大; 使图像明暗对比度提高, 导致标 准差更大；使图像熵值更大；晕光处一圈圈光军使反 映纹理细节的平均梯度变大; 晕光处明显的边界使边 缘强度更大; 导致空间域的活跃程度增大，空间频率 更大。不分区融合图像因包含晕光区的无用信息, 导 致客观评价结果失真。不分区指标数值变化无规律, 分区后, 由非晕光区指标能相对明显地看出融合图像 
的质量、算法的优劣等, 因此, 以非晕光区指标为研 究对象。

非晕光区数据中, IHS 变换的 EI 数值微大于 Curvelet 变换, 与主观评价矛盾, 因 IHS 变换的光谱 扭曲性使客观评价结果失真; $E$ 和 $\sigma$ 数值集中, 整体 变化趋势小, 对融合图像质量的区分度较小; $\bar{x} 、 \mathrm{AG}$ 、 $\mathrm{SF}$ 数值分布合理, 能明显表达融合图像的质量和不同
算法的优劣。

2) 融合图像对原始图像的信息保留度方面。评 价结果如表 3 所示。

从表 3 看出, RMSE 和 PSNR 数值整体变化小, 对算法优劣的区分度很小, 不适合作为评判指标; CE、 MI 数值相对分散, 能显著表达融合图像对原图信息 的保留度和不同算法的优劣。

\begin{tabular}{|c|c|c|c|c|c|c|}
\hline \multirow{2}{*}{ Algorithm } & \multicolumn{6}{|c|}{ No-halo region } \\
\hline & $\bar{x}$ & $\sigma$ & $E$ & AG & EI & SF \\
\hline IHS & 50.7814 & 22.9981 & 3.9085 & 1.5289 & 4.2002 & 8.1203 \\
\hline Curvelet & 61.8965 & 25.8810 & 3.9978 & 3.3561 & 4.0981 & 12.0089 \\
\hline IHS-Curvelet & 69.8815 & 28.9318 & 4.2760 & 3.6198 & 6.9078 & 15.6902 \\
\hline Improved IHS-Curvelet & 90.9179 & 29.9753 & 5.0362 & 3.8739 & 6.9201 & 17.8265 \\
\hline \multirow{2}{*}{ Algorithm } & \multicolumn{6}{|c|}{ No division } \\
\hline & $\bar{x}$ & $\sigma$ & E & $\mathrm{AG}$ & EI & SF \\
\hline IHS & 102.1417 & 25.9308 & 5.7862 & 3.9899 & 14.0576 & 10.1062 \\
\hline Curvelet & 105.318 & 38.7324 & 6.8762 & 6.8807 & 18.7757 & 21.1077 \\
\hline IHS-Curvelet & 106.8972 & 39.4403 & 7.0812 & 7.7245 & 20.8070 & 22.9812 \\
\hline Improved IHS-Curvelet & 104.9308 & 38.4334 & 6.6463 & 6.3063 & 15.0324 & 19.3287 \\
\hline
\end{tabular}

表 3 融合图像对原图信息的保留度

Table 3 Retained degree of fusion image to primary image

\begin{tabular}{lcccccccc}
\hline \multicolumn{1}{c}{ Algorithm } & $\mathrm{CE}_{\mathrm{FU}-\mathrm{VI}}$ & $\mathrm{CE}_{\mathrm{FU}-\mathrm{IR}}$ & $\mathrm{MI}_{\mathrm{FU}-\mathrm{VI}}$ & $\mathrm{MI}_{\mathrm{FU}-\mathrm{IR}}$ & $\mathrm{RMSE}_{\mathrm{FU}-\mathrm{VI}}$ & $\mathrm{RMSE}_{\mathrm{FU}-\mathrm{IR}}$ & PSNR $_{\mathrm{FU}-\mathrm{VI}}$ & PSNR $_{\mathrm{FU}-\mathrm{IR}}$ \\
\hline IHS & 1.0036 & 0.9989 & 1.0010 & 0.9982 & 32.8879 & 34.7968 & 53.9912 & 56.9124 \\
Curvelet & 0.5812 & 0.7630 & 1.8053 & 1.5363 & 29.6591 & 33.0928 & 58.8864 & 61.2431 \\
IHS-Curvelet & 0.3428 & 0.5469 & 1.9342 & 3.0870 & 22.7983 & 29.2204 & 59.9091 & 63.883 \\
Improved IHS-Curvelet & 0.2218 & 0.3501 & 2.8196 & 4.7868 & 25.6853 & 28.7192 & 60.1991 & 64.993 \\
\hline
\end{tabular}

3) 人眼视觉效果方面。评价结果如表 4 所示。

表 4 融合图像的视觉效果

Table 4 Visual effect of fusion image

\begin{tabular}{lccc}
\hline \multicolumn{1}{c}{ Algorithm } & SSIM $_{\mathrm{FU}-\mathrm{VI}}$ & SSIM $_{\mathrm{FU}-\mathrm{IR}}$ & \multicolumn{1}{c}{$Q^{\mathrm{AB} / \mathrm{F}}$} \\
\hline IHS & 0.4978 & 0.5010 & 0.3081 \\
Curvelet & 0.5681 & 0.6265 & 0.3798 \\
IHS-Curvelet & 0.5789 & 0.6316 & 0.4252 \\
Improved IHS-Curvelet & 0.5792 & 0.6401 & 0.5468 \\
\hline
\end{tabular}

从表 4 看出, SSIM ${ }_{\mathrm{FU}-\mathrm{VI}} 、 \mathrm{SSIM}_{\mathrm{FU}-\mathrm{IR}}$ 数值变化小, 对融合图像质量的区分度很小; $Q^{\mathrm{AB} / \mathrm{F}}$ 能显著表达融合 图像对边缘信息的保真度及不同算法的优劣。

通过对晕光区和非晕光区指标的对比与分析, 甄 选出晕光消除度 $D$ 、均值 $\bar{X}$ 、平均梯度 $\mathrm{AG}$ 、空间频 率 $\mathrm{SF}$ 、交叉熵 $\mathrm{CE}_{\mathrm{FU}-\mathrm{VI}}$. $\mathrm{CE}_{\mathrm{FU}-\mathrm{IR}}$ 、互信息 $\mathrm{MI}_{\mathrm{FU}-\mathrm{VI}} 、 \mathrm{MI}_{\mathrm{FU}-\mathrm{IR}}$ 、 边缘保持度 $Q^{\mathrm{AB} / \mathrm{F}}$ 等 9 种指标作为抗晕光融合图像的 评价体系。将 9 种指标数据绘制雷达图如图 4, 因 $\mathrm{CE}$
数值越小性能越好, 为方便分析由 $\mathrm{CE}^{-1}$ 表示。

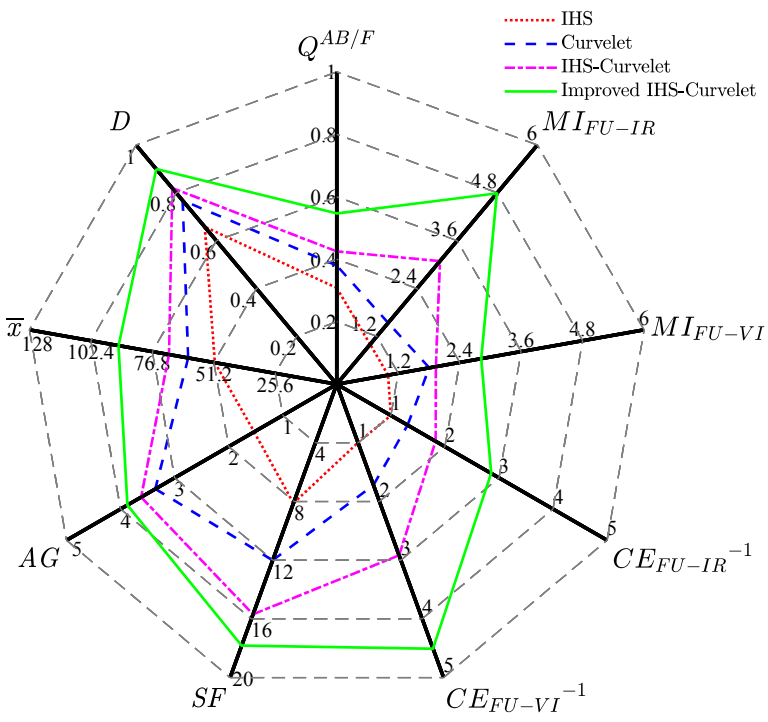

图 4 评价体系的雷达图

Fig.4 Radar chart of evaluation system 
由图 4 看出, 雷达图面积随算法的改进而增大, 9 种指标均能明显地反映融合图像的质量和不同算 法的优劣, 均表明 Curvelet 变换优于 IHS 变换; 在 Curvelet变换基础上使用 IHS 变换可进一步提高融合 图像的视觉效果; 将低频系数加权平均的 IHS-Curvelet 变换改为权值自动调节融合策略后又 可进一步改善融合图像的视觉效果, 与主观评价结 果一致。

\section{3 不同场景的评价结果}

为验证本文所甄选的抗晕光融合图像质量评价 体系对不同晕光场景的适用性, 采集市郊道路和市内 小区两组不同晕光场景的图像进行试验, 图 5 为市郊 开启远光灯约 $15 \mathrm{~m}$ 处的融合结果, 图 6 为市内开启 远光灯约 $20 \mathrm{~m}$ 处的融合结果。其中, 图 5 和图 6 中(a)、 (b)分别为原始可见光和红外图像，(c) (f) 分别为 IHS、 Curvelet、IHS-Curvelet 及改进 IHS-Curvelet 变换的融 合结果。

从图 5 和图的 6(c) (f)可以看出, 从左到右, 融 合图像的晕光消除效果越好, 车辆、背景和道路边缘 轮廓越清晰, 即 IHS、Curvelet、IHS-Curvelet 及改进 IHS-Curvelet 变换的融合结果依次变优。

在市郊、市内两种不同场景下，利用评价体系的 9 种指标进行评价, 绘制雷达图如图 7、图 8 所示。

从图 7〜图 8 中可以看出, 随算法改进, 雷达图包围 面积也越来越大, 9 种指标能从不同角度反映了抗晕 光融合图像质量的好坏及不同算法的优劣, 与主观评 价的结果一致, 验证了本文提出的抗晕光融合图像质 量评价体系具有一定的普适性。

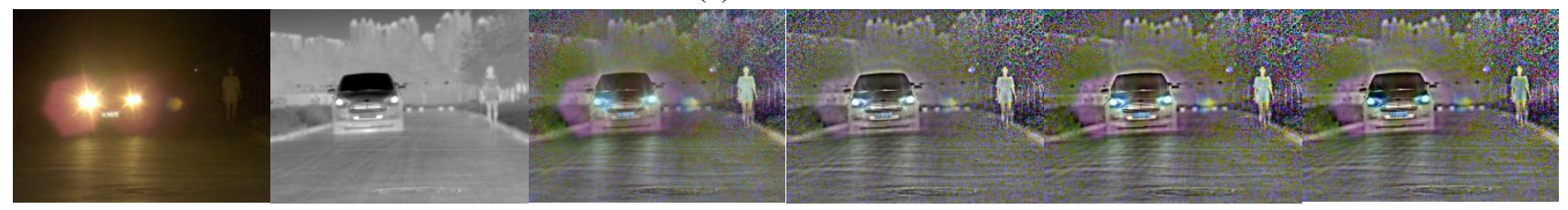
(a) 可见光图像
(b) 红外图像
(c) IHS 变换
(d) Curvelet 变换
(e) IHS-Curvelet
(f) 改进 IHS-Curvelet

$\begin{array}{lll}\text { (a) Visible image } & \text { (b) Infrared image } & \text { (c) IHS transform }\end{array}$

(d) Curvelet transform

(e) IHS-Curvelet

(f) Improved IHS-Curvelet

图 5 市郊道路约 $15 \mathrm{~m}$ 处的融合图像

Fig.5 Fusion image of suburban road at about $15 \mathrm{~m}$

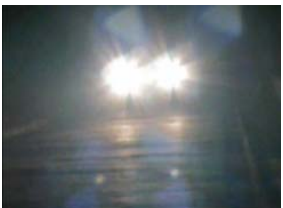

(a) 可见光图像

(a) Visible image

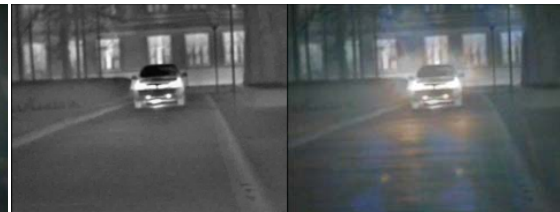

(b) 红外图像

(b) Infrared image (c) IHS 变换

(c) IHS transform

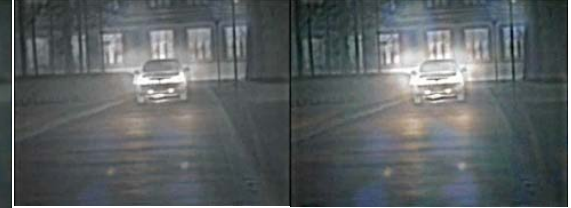

(d) Curvelet 变换

(e) IHS-Curvelet

\section{(f) 改进 IHS-Curvelet

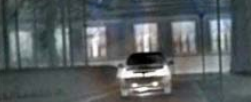

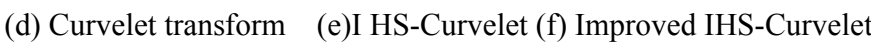

图 6 市内小区约 $20 \mathrm{~m}$ 处的融合图像 Fig.6 Fusion image of city community at about $20 \mathrm{~m}$

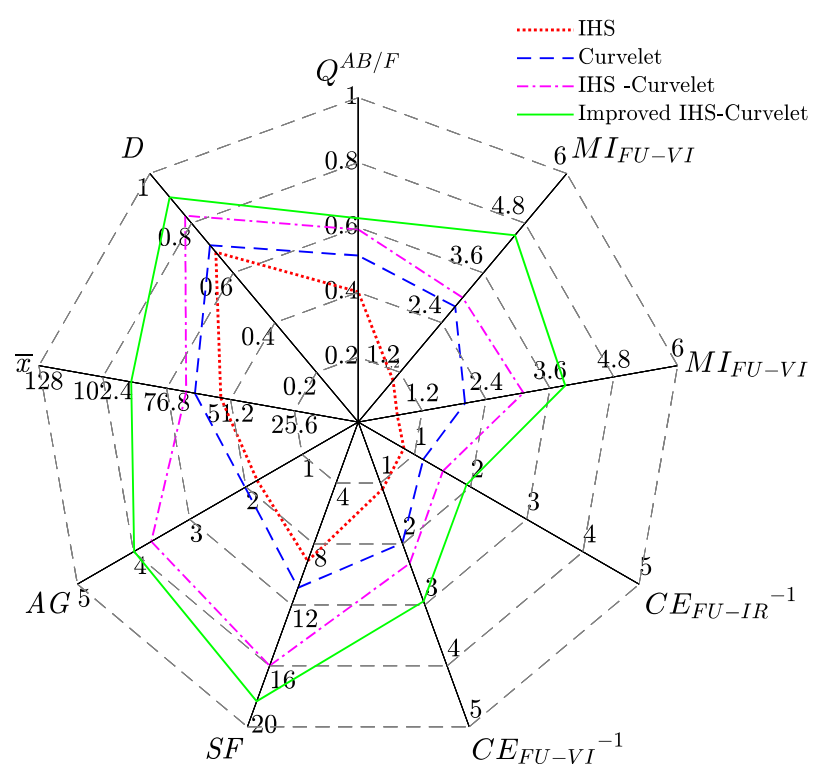

图 7 市郊道路约 $15 \mathrm{~m}$ 处的雷达图

Fig.7 Radar chart of suburban road at about $15 \mathrm{~m}$

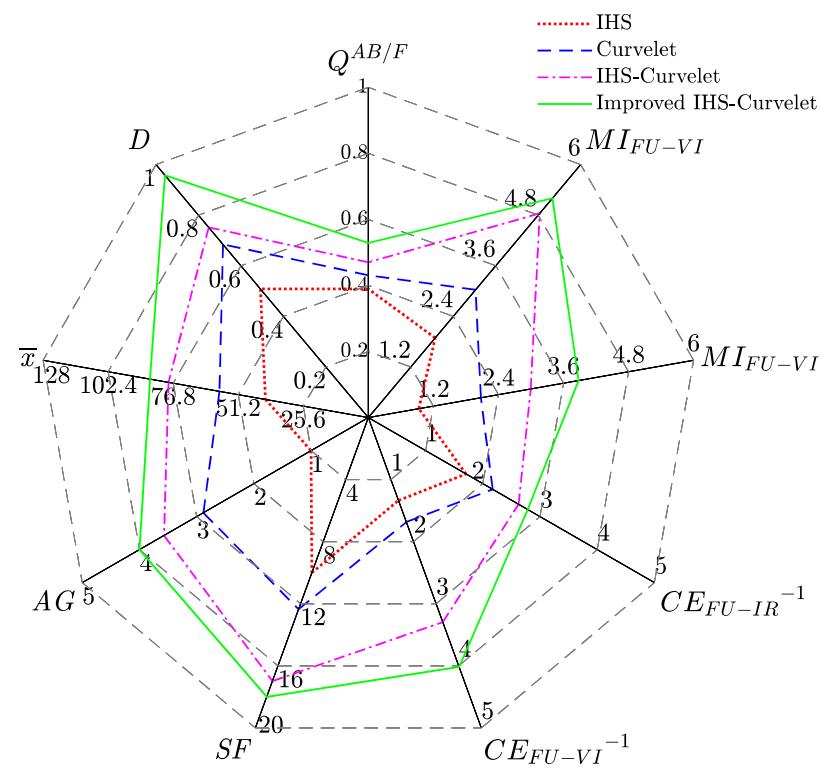

图 8 市内小区约 $20 \mathrm{~m}$ 处的雷达图

Fig.8 Radar chart of city community at about $20 \mathrm{~m}$ 


\section{4 结论}

针对现有的可见光与红外融合图像评价方法不 适用于评价汽车抗晕光融合图像的问题, 本文方法解 决了汽车抗晕光融合图像的客观评价结果与人眼视 觉效果不一致的问题, 克服了融合图像晕光消除越彻 底评价结果反而越差的缺点, 也适用于评判不同可见 光与红外融合的抗晕光算法的优劣。本文设计的自适 应迭代阈值法, 根据可见光图像的晕光程度自动确定 可见光灰度图像的晕光临界灰度值, 并将融合图像自 动分为晕光区和非军光区; 在晕光区设计晕光消除度 指标评价融合图像晕光消除的效果; 在非晕光区从多 角度评价融合图像色彩、细节信息的增强效果。甄选 出 9 种指标构成完整的图像质量评价体系, 全面、合 理地评价可见光与红外融合的汽车抗晕光融合图像。

\section{参考文献:}

[1] 陈琳.夜间行车远光灯会车法律问题研究 [J]. 法制与社会, 2014(23): $71-72$.

CHEN Lin. The legal issues study on the high beams car meeting at night[J]. Legal System and Society, 2014(23): 71-72.

[2] Marsell E, Bostrom E, Harth A. Spatial control of multiphoton electron excitations in InAs nanowires by varying crystal phase and light polarization[J]. Nano Letters, 2018, 18(2): 907-915.

[3] Chrzanowski, K. Review of night vision technology[J]. Opto-electronics Review, 2013, 21(2): 153-181.

[4] Kwak J Y, Ko B C, Nam J Y. Pedestrian tracking using online boosted random ferns learning in far infrared imagery for safe driving at night[J]. IEEE Transactions on Intelligent Transportation System, 2017, 18(1): 69-81.

[5] Jeong M R, Kwak J Y, Son J E, et al. Fast pedestrian detection using a night vision system for safety driving $[\mathrm{C}] / 11$ th International Conference on Computer Graphics, Imaging and Visualization, Singapore, 2014: DOI: 10.1109/CGiV.2014.25.

[6] Bosiers J T, Kleimann A C, Van Kuijk H C, et al. Frame transfer CCDs for digital still cameras: concept, design and evaluation[J]. IEEE Transactions on Electron Devices, 2002, 49(3): 377-386.

[7] 王健, 高勇, 雷志勇, 等. 基于双 CCD 图像传感器的汽车抗晕光方法 研究[J]. 传感技术学报, 2007(5): 1053-1056.

WANG Jian, GAO Yong, LEI Zhiyong, et al. Research of auto anti-blooming method based on double CCD image sensor[J]. Chinese Journal of Sensors and Actuators, 2007(5): 1053-1056.
[8] 郭全民, 李晓玲. 基于可见光和红外图像融合的汽车抗晕光方法 $[\mathrm{J}]$. 红外技术, 2015, 37(6): 475-478.

GUO Quanmin, LI Xiaoling. Vehicles' anti-blooming method based on visible and infrared images fusion[J]. Infrared Technology, 2015, 37(6): 475 - 478.

[9] 王晓红, 卢辉, 麻祥才, 等. 不同亮度下无参考图像质量评价方法[J]. 光学技术, 2018, 44(5): 569-575.

WANG Xiaohong, LU Hui, MA Xiangcai, et al. No-reference image quality assessment method for different ambient illumination[J]. Optics Technology, 2018, 44(5): 569-575.

[10] LI L, XIA W, LIN W, et al. No-reference and robust image sharpness evaluation based on multiscale spatial and spectral features[J]. IEEE Transactions on Multimedia, 2017, 19(5): 1030-1040.

[11] Jain A, Bhateja V. A full-reference image quality metric for objective evaluation inspatial domain[C]/12th International Conference on Communication and Industrial Application, 2011: DOI: 10.1109/ ICCIndA.2011.6146668.

[12] CHEN Guo, LI Li, JIN Weiqi. Image contrast enhancement method based on display and human visual system characteristics[J]. Applied Optics, 2019, 58(7): 1813-1823.

[13] 郭全民, 王言, 李翰山. 改进 IHS-Curvelet 变换融合可见光与红外图 像抗晕光方法[J]. 红外与激光工程, 2018, 47(11): 440-448.

GUO Quanmin, WANG Yan, LI Hanshan. Anti-halation method of visible and infrared image fusion based on improved IHS-Curvelet transform[J]. Infrared and Laser Engineering, 2018, 47(11): 440-448.

[14] 杨艳春, 李娇, 王阳萍. 图像融合质量评价方法研究综述 [J]. 计算机 科学与探索, 2018, 12(7): 1021-1035.

YANG Yanchunn, LI Jiao, WANG Yangping. Review of image fusion quality evaluation methods[J]. Computer Science and Exploration, 2018, 12(7): 1021-1035.

[15] 姚旺, 刘云鹏, 朱昌波. 基于人眼视觉特性的深度学习全参考图像质 量评价方法[J]. 红外与激光工程, 2018, 47(7): 39 -46.

YAO Wang, LIU Yunpeng, ZHU Changbo. Deep learning of full -reference image quality assessment based on human visual properties[J]. Infrared and Laser Engineering, 2018, 47(7): 39-46.

[16] 郭全民, 董亮, 李代娣. 红外与可见光图像融合的汽车抗晕光系统[J]. 红外与激光工程, 2017, 46(8): 171-176.

GUO Quanmin, DONG Liang, LI Daidi. Vehicles anti-halation system based on infrared and visible images fusion[J]. Infrared and Laser Engineering, 2017, 46(8): 171-176. 